\title{
Belträge zur Kenntnis des Bilirubins und Hämins.
}

\author{
Von
}

\section{William Küster.}

(Aus dem chemischen Laboratorium der Tierärztl. Hochschule in Stuttgart.)

(Der Redaktion zugegangen am 24. November 1912.)

Die Veröffentlichung von H. Fischer und F. MeyerBetz $\left.{ }^{1}\right)$ über die Porphyrinbildung gibt mir Veranlassung, einige Beobachtungen zu publizieren, die ich bis zur völligen Sicherstellung mit Hilfe größerer Materialmengen zurückgestellt hatte.

H. Fischer hat bei der Oxydation des Mesophorphyrins neben Hämatinsäure Methyläthylmaleinimid erhalten und damit das von mir in Gemeinschaft mit Herrn Deihle erübrigte Resultat bestätigt. ${ }^{2}$ ) Aus diesem habe ich den Schluß gezogen, daß im Hämin zwei Vinyle vorhanden sind, die bei der durch Reduktion erfolgenden Überführung in Mesoporphyrin «Äthyle* geben und damit den Pyrrolkomplex des Mesoporphyrins, der sie trägt, so widerstandsfähig machen, daß er bei der Oxydation als Imid erhalten bleibt.

H. Fischer glaubt nun, daß die Anwesenheit von Vinylen noch nicht sicher erwiesen sei, weil die durch Einwirkung von Natriumamalgam auf $\mathrm{Hämin}^{8}$ ) entstehende Leukoverbindung bei der Oxydation kein Imid gebe. Er setzt also voraus, daß die gelinde Reduktion des Hämins durch Jodwasserstoff, welche zum Mesoporphyrin führt, und die Reduktion durch Natriumamalgam in bezug auf die Vinyle gleichen Effekt haben müsse, und begründet diese Ansicht damit, daß das von ihm entdeckte Hemi-

1) Diese Zeitschrift, Bd. 82, S. 96 (1912).

2) Wir haben das Resultat inzwischen insofern vervollständigt, als wir von analysenreinem Mesoporphyrin ausgegangen sind, aber die sofortige Veröffentlichung unterlassen zu können geglaubt, weil wir nach unserer ersten Notiz der ungestörten Weiterbearbeitung der erzielten Befunde sicher zu sein wähnten.

$\left.{ }^{3}\right)$ Nach meiner Interpretation der Hämatoporphyrinbildung (Ber. d. d. chem. Ges., Bd. 45, S. 1935 (1912) wird die Leukoverbindung dieses Körpers von vornherein als zur Bildung des Imids bei der Oxydation nicht befähigt anzusprechen sein.

Hoppe-Seyler's Zeitschrift f. physiol. Chemie. LXXXII. 
bilirubin, das aus Bilirubin durch Reduktion mit Natriumamalgam entsteht, bei der Oxydation neben Hämatinsäure das Imid liefert, während er aus Bilirubin selbst nur die letztere erhielt. ${ }^{1}$ )

Demgegenüber verweise ich auf die schöne Arbeit von H. Emde, ${ }^{2}$ ) wonach bei der Einwirkung von Natriumamalgam auf Phenyläthylammoniumverbindungen Styrol, bei der Aufsprengung des reduzierten Isochinolinrings durch Natriumamalgam 0 -Vinylbenzyldimethylanilin und o-Methylstyrol entstehen:

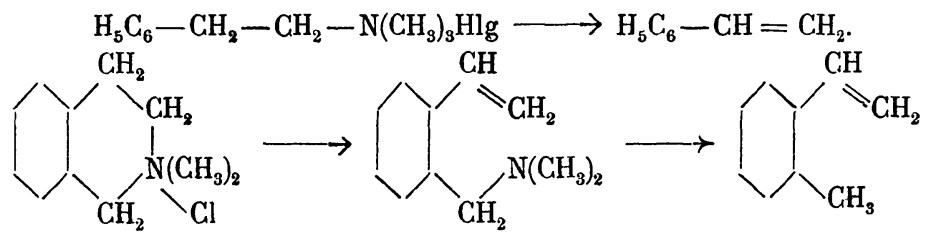

woraus gefolgert werden kann, daß Natriumamalgam vorhandene Vinyle nicht anzugreifen braucht. Bei der Überführung von Hämin in die Leukoverbindung, wie bei der Reduktion des Bilirubins zum Hemibilirubin können also die Vinyle erhalten bleiben und, wenn das letztere bei der Oxydation trotzdem das «Imid» gibt, so bleibt als einzige Möglichkeit die Annahme übrig, daß bereits im Bilirubin selbst ein Komplex enthalten ist, der bei der Oxydation das Imid liefert. In der Tat hat uns nun die Oxydation des Bilirubins das gesuchte Imid gegeben, wie ich bereits auf der Naturforscherversammlung in Karlsruhe mitteilte, allerdings in sehr geringer Menge, und ich habe darauf hingewiesen, daß mit diesem Befunde die Tatsache im besten Einklang steht, wonach aus Hämin zwei Moleküle Hämatinsäure entstehen, aus Bilirubin dagegen nur ein Molekül Hämatinsäure zu erhalten ist. Von den beiden Komplexen des Hämins, die bei der Oxydation diese Säure liefern:

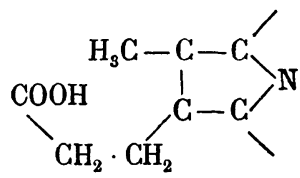

1) Diese Zeitschrift, Bd. 75, S. 339 (1911).

$\left.{ }^{2}\right)$ Liebigs Ann. der Chem., Bd. 391, S. 88 (1912). 
muß also der eine beim Übergang in Bilirubin Kohlendioxyd verloren haben und liefert jetzt bei der Oxydation das «Imid *

Als eine weitere Stütze für diese Ansicht, wonach bei der Bildung des Bilirubins aus Hämatin eine Abspaltung von Kohlendioxyd eingetreten ist, führe ich an, daß die Veresterung des Bilirubins durch Salzsäure und Methylalkohol anders verläuft wie beim Hämin oder Hämatoporphyrin. Auch aus dem Bilirubin wird ein Dimethylderivat gebildet, aber aus den Analysen dieses Esters geht mit großer Wahrscheinlichkeit hervor, daß ein Ersatz von Wasserstoff durch Methyl nur einmal erfolgt, das zweite Methyl wird durch Einlagerung von Methylalkohol aufgenommen. Ferner wird dem Hemibilirubin durch das Auffinden einer in Chloroform leicht löslichen "aciden Form» des Bilirubins, die sich in Natriumbicarbonat löst, eine bisher gegenüber dem Bilirubin als charakteristisch angesehene Eigenschaft genommen, so daß ihm als wesentlich unterscheidendes Merkmal abgesehen von der Farbe nur die Fähigkeit verbleibt, komplexe Salze zu bilden und mit p-Dimethylaminobenzaldehyd ein Kondensationsprodukt zu geben.

Schließlich kann ich nicht umhin, Piloty entgegenzutreten, wenn er, die biochemische Bildung des Bilirubins ganz unbeachtet lassend, ${ }^{\text {) }}$ das Entstehen des Bilirubins als durch einen synthetischen Prozeß hervorgerufen in Frage ziehen zu müssen und bejahen zu können glaubt. ${ }^{2}$ ) Scheut er sich doch sogar nicht, eine Parallele zwischen Eiweiß- und Bilirubinbildung im Organismus zu ziehen, also die für das Leben

1) Z. B. die Bildung des Hämatoidins von Virchow. Nach Brugsch (Z. f. exp. Path. u. Ther., Bd. 8, S. 645 (1911); Chem. C. B., 1911/1, S. 995) entgeht Hämatoporphyrin nach der Einverleibung der Bildung von Gallenfarbstoff, was nach meiner Auffassung von der Konstitution dieses Körpers durchaus verständlich wird. Leider gebraucht $\mathrm{Brugsch}$ die veralteten Formeln Nenckis, nach denen Hämatoporphyrin und Bilirubin als isomere Körper erscheinen.

2) Liebigs Ann., Bd. 390, S. 92 (1912): «die Entstehung des Bilirubins erfolgt wahrscheinlich nicht durch direkte Umwandlung des Blutfarbstoffs, sondern durch teilweisen Abbau desselben und Wiederaufbau des neuen Gebildes aus den passend veränderten Bruchstücken des Blutfarbstoffs. 2 
wichtigsten Körper mit einem Produkt $z u$ vergleichen, das er sich selbst aus Konkrementen, also pathologischen Gebilden dargestellt hat.

Dieser falsche Gedanke entspringt dem Wunsch, dem Entstehen seiner "Bilinsäure» einen höheren Wert für die $\mathrm{Zu}$ sammengehörigkeit von Bilirubin und Hämatin beimessen zu können, als dem Entstehen der Hämatinsäure aus beiden Farbstoffen, weil sie ein größeres Stück des Moleküls vorstellt als die Hämatinsäure, wobei Piloty auch noch voraussetzen muß, daß seine Bilinsäure $\mathrm{C}_{17} \mathrm{H}_{26} \mathrm{O}_{3} \mathrm{~N}_{2}$ und seine Hämatopyrrolidincarbonsäure $\mathrm{C}_{17} \mathrm{H}_{26} \mathrm{O}_{2} \mathrm{~N}_{2}$ denselben Aufbau haben und sich nur durch den Sauerstoffgehalt unterscheiden, während nicht einmal die empirische Formel der letztgenannten Säure sicher erwiesen ist ${ }^{1)}$ und Piloty ${ }^{2}$ ) neuerdings selbst zugeben muß, daß in ihr ein Gemisch vorliegt. Nun entsteht die Bilirubinsäure ${ }^{3}$ ) aus Bilirubin durch eine gemäßigte Reduktion mittels Jodwasserstoff und Jodphosphonium, sie ist also am ehesten dem durch ein gleiches Verfahren aus dem Hämin entstehenden Mesoporphyrin zu vergleichen, bei nachfolgender Oxydation liefern dann auch beide Körper Hämatinsäure und das Methyläthylmaleinimid. Die Reduktion erstreckt sich also auch im Bilirubin auf Vinyle; während aber im symmetrisch gebauten Hämin der Zusammenhang der vier Pyrrolkomplexe und damit der Charakter als Farbstoff erhalten bleibt, wird aus dem unsymmetrisch gebauten Bilirubin die farblose Bilirubinsäure herausgeschält. Auch sie zeigt also zunächst lediglich durch die bei ihrer 0xydation gewonnenen Spaltstücke die nahe Verwandtschaft zwischen Bilirubin und Hämatin, und es kommt auf ihre Konstitution an, um den Weg zu erkennen, der vom Hämatin zum Bilirubin geführt hat. Daß hier die Interpretation der Versuche durch H. Fis cher weit glücklicher gewesen ist, als die Formulierung durch Piloty,

2) Vgl. H. Fischers Habilitationsschrift, München 1912, Seite 13 (Univers.-Buchdr. Dr. C. Wolf-Sohn).

2) Ber. d. Deutsch. chem. Ges., Bd. 45, S. 2595 (1912).

$\left.{ }^{3}\right)$ Die Bilinsäure Pilotys dürfte mit H. Fischers Bilirubinsäure (Ber. d. Deutsch. chem. Ges., Bd. 45, S. 1579, 1912) identisch sein. Da letztere zuerst beschrieben wurde, muß der Name «Bilirubinsäures für den neuen Körper beibehalten werden. 
die weder die Bildung des Imids noch die Bildung der Hämatinsäure ungezwungen zu erklären imstande ist, mag ein Blick auf die Bilder der Bilirubinsäure, resp. Bilinsäure veranschaulichen.<smiles>CCC1=C(C)C(Oc2[nH]c(C)c(CCC(=O)O)c2C)C1C</smiles>

Bilirubinsäure $\mathrm{C}_{18} \mathrm{H}_{24} \mathrm{O}_{3} \mathrm{~N}_{2}$.

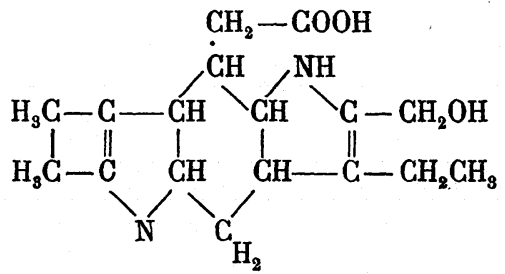

Bilinsäure $\mathrm{C}_{17} \mathrm{H}_{28} \mathrm{O}_{8} \mathrm{~N}_{2}$.

Allerdings muß nach der Beobachtung Pilotys, ${ }^{1}$ ) daß aus Bilirubinsäure durch Oxydation unter Wegnabme zweier Wasserstoffatome ein gefärbtes Produkt entsteht, auch die Fischersche Formel eine Änderung erfahren. Das Wesentliche in diesem Bilde besteht zurecht, namentlich die Nichtverknüpfung der beiden Pyrrolkomplexe durch ein Kohlenstoffatom. Dieser Gegensatz zum Hämin ist nämlich auch den Resultaten bei der Reduktion zu entnehmen, denn während Hämin Phonopyrrolcarbonsäure $^{2}$ ) und Isophonopyrrolcarbonsäure liefert, gibt Bilirubin nur die letztere.

1) Ber. d. Deutsch. chem. Ges., Bd. 45, S. 2393.

2) Nachdem sich das Phonopyrrol Pilotys als ein Kunstprodukt erwiesen hat, das im rohen Hämopyrrol nicht vorkommt, fallen die Gründe, die seinerzeit Piloty veranlaßt haben, den zuerst für diese Säure gewählten Namen sHämopyrrolcarbonsäure» in Phonopyrrolcarbonsäure zu ändern, fort, und man sollte sie mit dem ursprünglichen Namen wieder bezeichen, der auch das Verhältnis zum Hämopyrrol (2,3-Dimethyl4-äthylpyrrol) treffend beleuchtet; die Isophonopyrrolcarbonsäure würde dann im Anschluß an die von H. Fischer gewählte Bezeichnung Kryptopyrrol für das 3,5-Dimethyl-4-äthylpyrrol Kryptopyrrolcarbonsäure genannt werden können. 
<smiles>[R]CC1=NC(C[R])C(CCc2c[nH]c(C)c2C)C1CCC(=O)O</smiles>
im Hämin<smiles>[R]CC1N=C([R])C(C)C1CCC(=O)O</smiles>
im Bilirubin

Demnach fehlt dem Bilirubin dem Hämin gegenüber nicht nur ein Kohlenstoffatom, das sich, wie wir sahen, als Kohlendioxyd abgespalten hat, sondern noch ein zweites in Übereinstimmung mit den üblichen empirischen Formeln, wonach Hämin 34, Bilirubin 32 Kohlenstoffatome besitzt.

Ich erlaube mir aber in den Wettstreit um die geeignetste Formulierung der Bilirubinsäure einzugreifen, weil ich bereits im Jahre 1907 mit meinem Schüler Lacour zusammen ${ }^{1}$ ) ein Bild des Aufbaus des Häminmoleküls entworfen habe, das sich als nützlich erwiesen hat, ${ }^{2}$ ) wie sich $\mathrm{z}$. B. auch die Formulierung H. Fischers diesem Bilde anpaßt. Es ist vor der Entwirrung der Bestandteile des rohen Hämopyrrols aufgestellt worden, trägt also der Isomerie des Hämo- und Kryptopyrrols und der Konstitution des Phyllopyrrols als 2,3,5-Trimethyl-4-äthylpyrrols keine Rechnung. Auch die Existenz der isomeren Hämopyrrolcarbonsäuren, $\mathrm{zu}$ denen sich wohl noch ein 2,3,5-Trimethyl4-carboxyäthylpyrrol gesellen wird, ${ }^{3}$ ) ist nicht berücksichtigt. Ebensowenig das Vorhandensein von Vinylen. Und dennoch ändern sich auch jetzt nur einige Züge des Bildes, die den Gesamtcharakter zu verwischen nicht imstande sind. Ich lasse daher das Bild jetzt in seiner neuen Gestalt folgen und möchte nur noch erwähnen, daß wir zur Prüfung seiner Naturtreue

1) Dissertation Würzburg, J. Beckers Univers.-Buchdruckerei. Vgl. auch Ber. d. Deutsch. pharm. Ges., Bd. 21, S. 513 (1911).

2) Vgl. Willstätter, Liebigs Ann., Bd. 371, S. 33 (1909).

3) Vielleicht ist die sehr unbeständige Xanthopyrrolcarbonsäure Pilotys dieses tetrasubstituierte Pyrrol. 
weitere Versuche unternehmen werden. Bereits hat sich eine Addition von Brom an das Dimethylhämin als möglich erwiesen, und bin ich mit Herrn Greiner beschäftigt, die bei der Oxydation eines Dibromdimethylhämins entstehenden Produkte zu studieren.

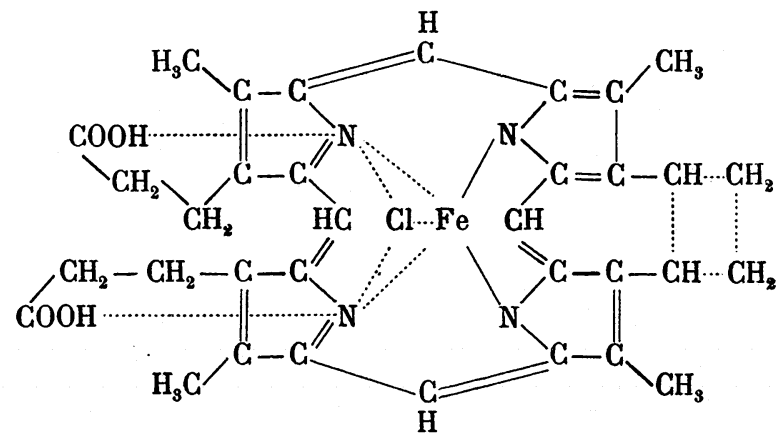

Hämin $\left.\mathrm{C}_{34} \mathrm{H}_{38} \mathrm{O}_{4} \mathrm{~N}_{4} \mathrm{FeCl} .{ }^{1}\right)$

Experimenteller Teil.

A. Über das Mesoporphyrin.

(Mitbearbeitet von Herrn P. Deihle.)

Die Darstellung des rohen Mesoporphyrins erfolgte wieder nach den Angaben Zaleskis, die Reinigung geschah nach den bereits in der ersten Mitteilung gemachten Angaben dadurch, $\mathrm{da} B$ das in kalter Natriumbicarbonatlösung (40:1500 Wasser) gelöste und mit Essigsäure wieder gefällte Rohprodukt aus $8 \mathrm{~g}$ Hämin mit $100 \mathrm{ccm} 10 \%$ iger Schwefelsäure auf dem Wasserbade 10 Minuten digeriert wurde, ${ }^{8}$ ) worauf die Lösung

1) Vgl. zu diesem Bilde meine Ausführungen über die Konstitution des Hämins und des Dimethylhämins: Ber. d. Deutscb. chem. Ges., Bd. 45, S. 1935̃, und Diese Zeitschrift, Bd. 82, S. 113 (1912).

2) Das hierbei Ungelöste wurde der gleichen Behandlung noch mehrere Male unterworfen, schließlich wurden auch die Mutterlaugen des salzsauren Mesoporphyrins durch Natriumpikrat gefällt und der erhaltene Niederschlag teils durch heißes Wasser, teils - nach dem Trocknen durch Petroläther von anhaftender Pikrinsäure befreit. Das nunmehr vorliegende amorphe Mesoporphyrin wurde dann mit $250 \mathrm{ccm}$ 2,5\% iger Salzsäure angerührt, die Lösung filtriert und durch Aufleiten von Chlorwasserstoff krystallisiertes salzsaures Salz zur Abscheidung gebracht. 
des schwefelsauren Mesoporphyrins abgesaugt und alsdann bei niederer Temperatur mit Chlorwasserstoff gesättigt wurde. Beim Stehen über Nacht setzte sich reichlich salzsaures Mesoporphyrin ab, das abgesaugt und mit kalter Salzsäure $\mathrm{SO}_{4}$-frei gewaschen wurde. Die Gesamtausbeute betrug 2,3 g, also $29 \%$ des verwendeten Hämins.

$$
\begin{array}{rccccc}
\text { Analyse. } & 0,1713 \text { Substanz : 0,3995 } \mathrm{CO}_{2} \text { und } 0,0966 \mathrm{H}_{2} \mathrm{O} \\
& 0,1312 & \text { Substanz : 0,0598 } & \mathrm{AgCl} . \\
\mathrm{C}_{34} \mathrm{H}_{38} \mathrm{O}_{4} \mathrm{~N}_{4} \cdot 2 \mathrm{HCl} & \text { Ber.: } 63,85 \% \mathrm{C} & 5,97 \% \mathrm{H} & 11,11 \% \mathrm{Cl} \\
& \text { Gef.: } 63,6 \% \mathrm{C} & 6,26 \% \mathrm{H} & 11,27 \% & \mathrm{Cl} .
\end{array}
$$

Die Oxydation des aus der Lösung von je 2,5 g des salzsauren Salzes mit Hilfe von Natriumacetat gefällten, abgesaugten und $\mathrm{Cl}^{\prime}$-frei gewaschenen Mesoporphyrins erfolgte in der Weise, daß der erhaltene Schlamm in $250 \mathrm{~g} 35 \%$ iger Schwefelsäure gelöst und die Lösung mit einer Auflösung von $3,6 \mathrm{~g}$ Chromtrioxyd in $30 \mathrm{~g}$ Wasser (12 Atome Sauerstoff auf ein Molekül Mesoporphyrin) versetzt wurde. Zur Einleitung der Reaktion wurde schwach erwärmt; nach dem Stehen über Nacht erwies sich die Chromsäure als verbraucht, worauf die Lösung erschöpfend mit Äther ausgeschüttelt wurde. Den ätherischen Auszügen wurde die Hämatinsäure durch Sodalösung entzogen, worauf sie getrocknet wurden. Nach Abdestillation des Äthers hinterblieben je $0,38 \mathrm{~g}$ Imid mit dem Schmelzpunkt $67^{\circ}$. Der Sodalösung wurde die Hämatinsäure nach dem Ansäuern ent\%ogen, ihre Menge betrug 0,59 resp. $0,68 \mathrm{~g}$, der Schmelzpunkt der daraus durch Verseifen gewonnenen Säure $\mathrm{C}_{8} \mathrm{H}_{8} \mathrm{O}_{3}$ lag bei $97^{\circ}$.

Es war also nach diesem Verfahren nicht ganz je eine Molekel der Oxydationsprodukte aus der Mesoporphyrinmolekel gewonnen worden.

Berechnet: 1,4 g Hämatinsäure und $11,09 \mathrm{~g}$ Imid.

Gefunden: 1,27 » »0,76 » »

Ich möchte noch bemerken, daß die Leichtlöslichkeit des Sulfats, die Schwerlöslichkeit des Chlorids so sehr an die Verhältnisse beim Hämatin erinnert, daß auch hieraus für das letztere die Mitbeteiligung der Stickstoffatome an der Salzbildung, für welche in erster Linie das Eisen in Betracht kommt, zu folgern ist. 


\section{B. Darstellung von Salzen und der aciden* Form des Bilirubins.}

In einer früheren Mitteilung ${ }^{1}$ ) habe ich bereits darauf hingewiesen, daß das Bilirubin in zwei Modifikationen besteht, von denen die in Chloroform leichter lösliche beim Aufbewahren in die schwerer lösliche übergeht. Als Grund dieser Veränderung glaubte ich eine sich allmählich vollziehende Polymerisation annehmen $z u$ dürfen. Daß daneben auch noch eine andere Veränderung des Moleküls, eine Wanderung von Wasserstoff etwa im Sinne einer Keto-Enol-Umlagerung sich vollzieht, glaubte ich bereits aus der Analyse von Silbersalzen verschiedener Bilirubinpräparate folgern zu können. Herr R. Schmiedel konnte alsdann die diesbezüglichen Resultate bestätigen, $d . \mathbf{h}$. es war möglich, fast vier Atome Silber in die Molekel Bilirubin einzuführen, wenn ein frisch aus Dimethylanilin umkrystallisiertes Präparat verwendet wurde, während ältere Präparate nur ungefähr zwei Atome Silber aufnahmen.

Die Darstellung dieser Silbersalze erfolgte durch Fällen der sehr verdünnten Lösungen der Kalium- oder Natriumsalze der betreffenden Bilirubinpräparate, die mit Hilfe des Alkalis in einer zwei Molekeln meist etwas übersteigenden Menge hergestellt worden waren, durch Silbernitrat oder -sulfat. Die entstandenen Niederschläge waren zuerst rostfarben, färbten sich aber, trotzdem immer mit ausgekochtem Wasser gearbeitet wurde, schnell dunkel und erschienen nach dem Auswaschen und Trocknen im Vakuum unter Lichtabschluß als metallisch glänzende Stücke. Ich veranschauliche die Darstellung und die Ergebnisse der Analysen durch folgende Tabelle:

Analytische Belege. $\left.{ }^{2}\right)$

1. 0,2195 Subst.: $\quad 0,3519 \mathrm{CO}_{2}, 0,0793 \mathrm{H}_{2} \mathrm{O}$ und $0,0709 \mathrm{Ag}$.

2. $0,1535 \gg\left(120^{\circ}\right): 0,2454 \mathrm{CO}_{2}, 0,0581 \mathrm{H}_{2} \mathrm{O} \gg 0,0503 \mathrm{Ag}$.

3. 0,2284 > $\left(115^{\circ}\right): 0,3654 \mathrm{CO}_{2}, 0,0800 \mathrm{H}_{2} \mathrm{O}$ 》0,0741 Ag.

1) Diese Zeitschrift, Bd. 47, S. 294 (1906).

$\left.{ }^{2}\right)$ Die unter Nr. 1, 6, 7, 8 und 10 aufgeführten Analysen sind von Herrn R. Schmiedel, die andern von mir und meinem Assistenten J. Eppler bereits 1909 ausgeführt worden. 


\begin{tabular}{|c|c|c|c|c|c|c|c|c|c|c|}
\hline 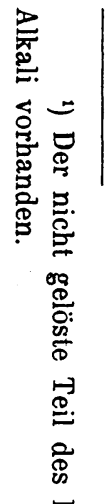 & ॰ & $\begin{array}{l}0 \\
*\end{array}$ & $\infty$ & 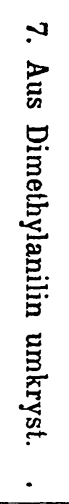 & 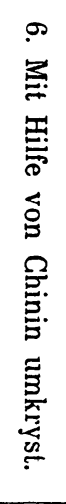 & $\begin{array}{l}e r \\
+ \\
: \\
\vdots \\
: \\
: \\
\vdots \\
: \\
: \\
\end{array}$ & 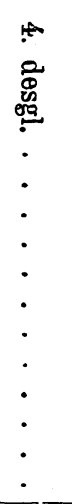 & 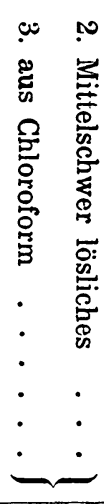 & 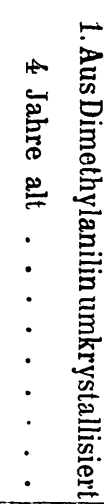 & 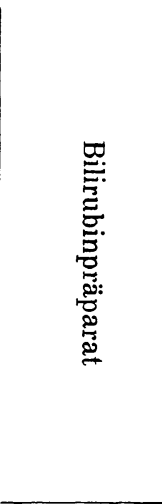 \\
\hline 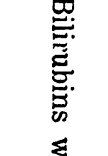 & $\frac{\mapsto}{0}$ & 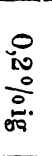 & $\frac{\overrightarrow{0}}{\frac{0}{0}}$ & $\frac{\overrightarrow{0}}{\stackrel{0}{\circ}}$ & $\frac{\stackrel{5}{0}}{\stackrel{5}{\circ}}$ & $\frac{\overrightarrow{0}}{\vdots}$ & $\frac{\overrightarrow{0}}{\stackrel{0}{0}}$ & 竞. & $\frac{\overrightarrow{0}}{\grave{0}}$ & 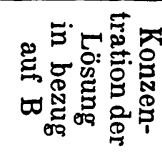 \\
\hline 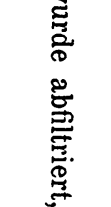 & $\begin{array}{l}\frac{N}{2} \\
= \\
=\end{array}$ & $\begin{array}{l}0 \\
= \\
=\end{array}$ & 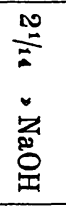 & $\begin{array}{l}\frac{0}{2} \\
\frac{1}{2} \\
0 \\
0\end{array}$ & $\begin{array}{l}\frac{2}{2} \\
= \\
0\end{array}$ & $\begin{array}{l}2 \\
0 \\
0 \\
0\end{array}$ & $\begin{array}{l}\frac{0}{5} \\
\frac{0}{3} \\
0 \\
0\end{array}$ & $\begin{array}{l}0 \\
5 \\
=\end{array}$ & 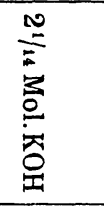 & 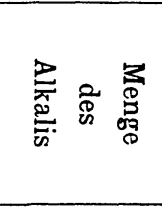 \\
\hline 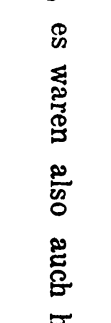 & 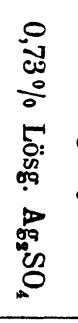 & 営 & & 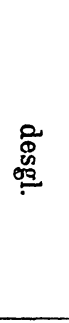 & 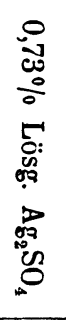 & 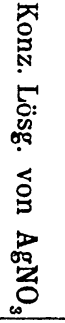 & 骂 & 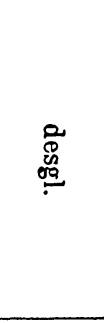 & 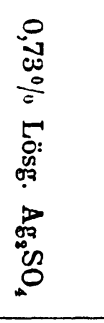 & 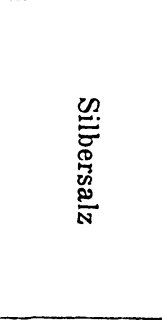 \\
\hline$\stackrel{8}{\sharp}$ & 沓. & $*$ & $\approx$ & $*$ & $*$ & $\bullet$ & $*$ & * & $\stackrel{+}{\dot{H}}$ & 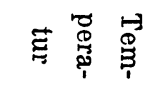 \\
\hline 胥 & 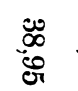 & $\stackrel{\infty}{\infty}$ & 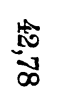 & $\begin{array}{l}\text { 空 } \\
+\infty\end{array}$ & 隹 & $\begin{array}{l}5 \\
8 \\
8\end{array}$ & 庶 & 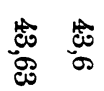 & $\stackrel{\vec{\omega}}{\vec{\omega}}$ & $\begin{array}{l}\circ \\
0\end{array}$ \\
\hline 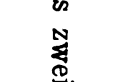 & $\underset{\sim}{\infty}$ & $\stackrel{\leftrightarrow}{\circ}$ & $\infty_{\infty}^{\infty}$ & $\stackrel{\Delta}{0}$ & $\stackrel{\leftrightarrow}{\sim}$ & \& & مِ & 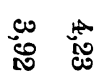 & 偪 & : \\
\hline $\begin{array}{l}\frac{3}{0} \\
\frac{0}{0} \\
\frac{0}{0}: \\
\frac{1}{0}\end{array}$ & $\stackrel{ث}{\rightleftarrows}$ & 今 & 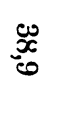 & $\begin{array}{l}\infty \\
\infty \\
\infty\end{array}$ & $\stackrel{\mathscr{\varpi}}{\mathscr{H}}$ & 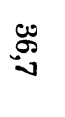 & 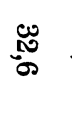 & 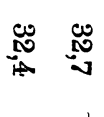 & W. & $\stackrel{0}{0}$ \\
\hline
\end{tabular}


4. 0,2483 Subst. $\left(110^{\circ}\right): 0,3958 \mathrm{CO}_{2}, 0,0878 \mathrm{H}_{2} \mathrm{O}$.

$0,2225 *\left(110^{\circ}\right): 0,0725 \mathrm{Ag}$.

5. 0,1966 > $\left(120^{\circ}\right): 0,2953 \mathrm{CO}_{2}, 0,0642 \mathrm{H}_{2} \mathrm{O}$.

$0,2145 \gg\left(120^{\circ}\right): 0,0787 \mathrm{Ag}$.

6. 0,1685 » $0,2643 \mathrm{CO}_{2}, 0,0561 \mathrm{H}_{2} \mathrm{O}$ und $0,0608 \mathrm{Ag}$.

7. $0,1665 » \quad: 0,2614 \mathrm{CO}_{2}, 0,0600 \mathrm{H}_{2} \mathrm{O} \gg 0,0640 \mathrm{Ag}$.

8. 0,2058 ": $0,3228 \mathrm{CO}_{2}, 0,070 \mathrm{H}_{2} \mathrm{O}$ " $0,080 \mathrm{Ag}$.

9. 0,2638 » $0,3685 \mathrm{CO}_{2}, 0,0752 \mathrm{H}_{8} \mathrm{O} \gg 0,1055 \mathrm{Ag}$.

10. $0,1982 »: 0,2830 \mathrm{CO}_{2}, 0,060 \mathrm{H}_{2} \mathrm{O} \gg 0,0815 \mathrm{Ag}$.

$\mathrm{Zu}$ diesen Analysen ist noch $\mathrm{zu}$ bemerken, daß das Trocknen bei höherer Temperatur nie eine merkliche $\mathrm{Ab}$ nahme gegenüber dem Vakuum gab, und daß verschiedene der Salze auf einen Kaliumgehalt untersucht worden sind, ohne daß ein solcher aufgefunden wurde.

Die Zusammensetzung der Silbersalze schwankt also stark und entspricht in den ermittelten Werten einem Gehalt von zwei bis zu vier Atomen Silber im Molekül des Bilirubins, indem sich berechnet für

$$
\begin{array}{lllll}
\mathrm{C}_{32} \mathrm{H}_{34} \mathrm{Ag}_{8} \mathrm{O}_{6} \mathrm{~N}_{4}: 48,9 \% & \mathrm{C} & 4,3 \% \mathrm{H} & 27,4 \% \mathrm{Ag} . \\
\mathrm{C}_{32} \mathrm{H}_{33} \mathrm{Ag}_{3} \mathrm{O}_{6} \mathrm{~N}_{4}: 43,0 \% \mathrm{C} & 3,8 \% \mathrm{H} & 36,2 \% \mathrm{Ag} . \\
\mathrm{C}_{32} \mathrm{H}_{32} \mathrm{Ag}_{4} \mathrm{O}_{6} \mathrm{~N}_{4}: 39,3 \% & \mathrm{C} & 3,2 \% \mathrm{H} & \mathbf{4 4 , 2} \% \mathrm{Ag} .
\end{array}
$$

Freilich muß dahingestellt bleiben, ob sich die Salze noch vom Bilirubin ableiten, da es nicht möglich war, unverändertes Bilirubin daraus wieder herzustellen. Das Silber erweist sich als recht fest gebunden; mit Alkalien geben die Salze kolloide Lösungen, eine Verdrängung des Silbers durch Baryum war nicht möglich, durch Calcium erfolgte sie zum Teil.

Das Silbersalz Nr. 6 wurde z. B. als Schlamm, d. h. frisch gefällt mit einer Lösung von Calciumchlorid geschüttelt, worauf filtriert und $\mathrm{Ca}^{\prime}$-frei gewaschen wurde. $0,3477 \mathrm{~g}$ der getrockneten Substanz gaben dann beim Veraschen: $0,0148 \mathrm{~g} \mathrm{CaO}$ $=3 \%$ Ca. Ein weiteres Silbersalz wurde noch von Herrn Schmiedel aus einem Kaliumsalz folgender Darstellungsart gewonnen:

$1 \mathrm{~g}$ Bilirubin wurde mit $250 \mathrm{ccm}$ Chloroform geschüttelt, der ungelöste Farbstoff abfiltriert und aus dem Filtrat das 
Lösungsmittel bis auf $50 \mathrm{ccm}$ abdestilliert. Diese konzentrierte Lösung wurde nun mit $15 \%$ iger Kalilauge geschüttelt, wobei sich das Kaliumsalz im festen Zustande abschied, sodaß die flüssigen Schichten abgegossen werden konnten, worauf das Salz auf einen Tonteller gebracht und durch Betupfen mit Wasser von anhängender Lauge befreit werden konnte. Die Ausbeuten aus mehreren Versuchen wurden vereint. $0,1344 \mathrm{KCl}$.

$0,602 \mathrm{~g}$ dieses Salzes gaben mit Salzsäure zersetzt $\mathrm{C}_{32} \mathrm{H}_{34} \mathrm{~K}_{2} \mathrm{O}_{6} \mathrm{~N}_{4}$. Berechnet: $12,03 \% \mathrm{~K}$. Gefunden: $11,7 \mathrm{~K}$.

$0,8 \mathrm{~g}$ dieses übrigens in Alkohol leicht löslichen Salzes gaben nun in $100 \mathrm{ccm}$ Wasser gelöst mit Silbersulfat einen Niederschlag folgender Zusammensetzung:

$$
41,24 \% \text { C } \quad 3,5 \% \text { H } \quad 40,8 \% \text { Ag. }
$$

0,2250 Substanz: 0,3402 $\mathrm{CO}_{2}, 0,0707 \mathrm{H}_{2} \mathrm{O}$ und 0,0920 Ag.

Es waren also auch hier fast vier Atome Silber eingetreten. Die Analyse stimmt merkwürdig genau auf ein äquimolekulares Gemisch der Salze mit 3 und 4 Atomen Silber.

Während das Silber sehr fest gebunden ist und sich durch Baryum nicht verdrängen läßt, wird letzteres sehr leicht durch Calcium ersetzt. $1 \mathrm{~g}$ des analog den Silbersalzen hergestellten Baryumsalzes - nach dem Trocknen metallisch glänzende Stückchen mit dem für die Formel $\mathrm{C}_{32} \mathrm{H}_{34} \mathrm{BaO}_{6} \mathrm{~N}_{4}$ berechneten Baryumgehalt von $19,3 \% 0^{1}$ ) - wurde mit einer Lösung von $0,31 \mathrm{CaCl}_{2}$ in $100 \mathrm{ccm}$ Wasser im Schüttelapparat längere Zeit behandelt, worauf filtriert und Ca-frei gewaschen wurde. Im Filtrat fand sich Bä, das unlösliche Salz enthielt nur noch Calcium und zwar in der berechneten Menge:

0,1278 Substanz: 0,0125 CaO.

Ber. f. $\mathrm{C}_{32} \mathrm{H}_{34} \mathrm{CaO}_{6} \mathrm{~N}_{4}: 6,56 \%$ Ca. Gef: $7,00 .{ }^{2}$ )

Das Zinksalz zeigte nach unseren Analysen keine normale Zusammensetzung. Unter den für die Darstellung des

1) 0,0762 g Subst.: 0,0180 g Asche $=0,0253 \mathrm{~g} \mathrm{BaSO}_{4}=19,4 \% \mathrm{Ba}$.

2) Ganz ähnliche Resultate ergaben sich ja beim Hämatin, Diese Zeitschrift, Bd. 66, S. 191 (1910). 
Silbersalzes eingehaltenen Bedingungen bekam Herr Sch miedel ein Salz, das bei der Analyse folgende Werte gab:

0,2106 Subst.: 0,4561 $\mathrm{CO}_{2}, 0,0764 \mathrm{H}_{2} \mathrm{O}$ und $0,0385 \mathrm{ZnO}$

$$
=59,2 \% \mathrm{Cu} \quad 4,03 \% \mathrm{H} \quad 14,6 \% \mathrm{Zn} \text {. }
$$

Ich habe dann noch ein Zinksalz aus alkoholischer Lösung hergestellt.

$1,4 \mathrm{~g}$ reines Bilirubin ${ }^{1}$ ) wurden mit $100 \mathrm{ccm} 90 \%$ igen Alkohols und $5 \mathrm{ccm}$ n-Natronlauge geschüttelt, vom Ungelösten $(0,5 \mathrm{~g})$ filtriert und das Filtrat mit einer alkoholischen Zinkacetatlösung im Überschuß versetzt. ${ }^{2}$ ) Der sehr schön rot gefärbte Niederschlag wurde darauf Zn-frei gewaschen und im Vakuum getrocknet, wobei er in grünschwarze, metallisch glänzende Stücke überging, die fein gepulvert eine braunrote Farbe hatten.

0,2288 Subst. $\left.\left(120^{\circ}\right)^{3}\right): 0,4833 \mathrm{CO}_{2}, 0,104 \mathrm{H}_{2} \mathrm{O}$ und $0,0330 \mathrm{ZnO}$

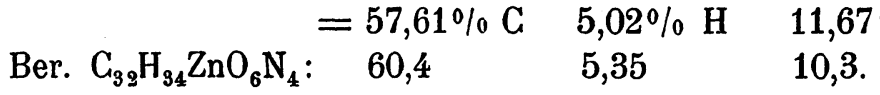

Das aus diesem Zinksalz mit Hilfe von Essigsäure wiederhergestellte Bilirubin hatte nun allerdings noch einen Aschengehalt von $2 \%$, gab aber bei der Analyse annähernd richtige Werte.

0,1495 Subst. $\left(110^{\circ}\right): 0,3581 \mathrm{CO}, \quad 0,0808 \mathrm{H}^{2} \mathrm{O}, 0,0029$ Asche 0,2058 * $\left(110^{\circ}\right): 17,2 \mathrm{ccm} \mathrm{N}$ bei $16,5^{\circ}$ und $746 \mathrm{~mm} \mathrm{~B}$.

Ber. $\mathrm{C}_{38} \mathrm{H}_{36} \mathrm{O}_{6} \mathrm{~N}_{4}: 67,1 \%$ C $6,3 \% \quad 9,7 \% \mathrm{~N}$

Gef.: $\left.\quad 66,626,12 \% \mathrm{H}^{4}\right) 9,54 \% \mathrm{~N}$

Mit diesem Befund wird die Angabe von Köster ${ }^{5}$ ) in seiner Dissertation bestätigt, daß aus dem Calciumsalz Bilirubin und $6,4 \% \mathrm{H}$.

1) 0,1442 g Subst. $\left(110^{\circ}\right): 0,3550$ g CO u. 0,0831 g H$_{8} \mathrm{O}=67,14 \% \mathrm{C}$

$0,1610 \mathrm{~g}$ Subst. $\left(110^{\circ}\right): 13,1 \mathrm{ccm} \mathrm{N}$ bei $18^{\circ}, 748 \mathrm{~mm}=9,24 \% \mathrm{~N}$.

2) Jedes Erwärmen ist zu vermeiden, da Zinkhydroxyd auch auf Bilirubin in alkalischer Lösung sehr schnell einwirkt, d. h. das Ergrünen der alkalischen Lösung tritt bei Gegenwart von Zink sehr viel schneller ein. Die Erscheinung soll verfolgt werden.

$\left.{ }^{3}\right)$ Beim Trocknen ging die rote Farbe in Braungrün über, die Abnahme betrug $0,0065 \mathrm{~g}$.

4) Auf aschefreie Substanz bezogen.

5) Rostock, C. Hinstorffs Buchdruckerei, 1901. 
regeneriert werden kann. Zugleich wurde dann aber die Beobachtung gemacht, daß sich das aus dem Zinksalz wiedergewonnene Bilirubin im Gegensatz zu dem verwendeten Bilirubin - die Proben wurden neben einander angestellt - in einer bei Zimmertemperatur bereiteten Lösung von saurem Natriumcarbonat wenigstens $\mathrm{zu}$ einem großen Teil auflöst. Hier muß also eine «aci»-Form des Bilirubins vorliegen, von der ich allerdings weitere unterscheidende Merkmale bisher nicht angeben kann. Wahrscheinlich ist diese Modifikation in Chloroform sehr viel leichter löslich als das gewöhnliche Bilirubin. Wenn man nämlich eine alkalische Lösung des Bilirubins ansäuert und die Suspension mit Chloroform ausschüttelt, so erhält man eine Konzentration, wie sie früher auch bei soeben umkrystallisiertem Bilirubin beobachtet worden ist. ${ }^{1}$ )

$18,72 \mathrm{~g}$ der Lösung enthielten 0,047 g Bilirubin. Löslichkeitsverhältnis $1: 397$.

19,35 g einer zweiten Lösung enthielten 0,0790 Bilirubin. Löslichkeitsverhältnis $1: 247$.

Die acide Form des Bilirubins wird auch noch aus dem Ammoniumsalz erhalten, das sich durch Leichtlöslichkeit in Methylalkohol auszeichnet.

$2 \mathrm{~g}$ Bilirubin wurden in $25 \mathrm{ccm} 90 \%$ igem Methylalkohol suspendiert und trocknes Ammoniak eingeleitet, worauf der Farbstoff sich sehr schnell und vollständig löst. Die filtrierte Lösung wurde im Vakuum verdampft, es hinterblieben fast schwarze Lamellen im Gewicht von $2 \mathrm{~g}$, die zerrieben ein braunrotes Pulver vorstellten, das an Wasser etwas Substanz abgab. Wenn hier eine Ammoniakverbindung vorliegt, so würde der bei $120^{\circ}$ eintretende Gewichtsverlust von $10,22 \%$ dafür sprechen, daß das Bilirubin 3-4 Moleküle Ammoniak zu addieren vermag.

0,3230 Substanz verloren bei $120^{\circ} 0,033 \mathrm{~g}$.

0,1266 Subst. $\left(120^{\circ}\right): 10,8 \mathrm{~cm} \mathrm{~N}$ bei $10^{\circ}, 749 \mathrm{~mm}=10,06 \% \mathrm{~N}$.

Nach letzterer Analyse zu urteilen, war das aufgenommene Ammoniak bei $120^{\circ}$ wieder vollständig abgegeben worden, es

1) Diese Zeitschrift, Bd. 47, S. 316 (1906). 
lag also wieder Bilirubin vor, aber in der «aci»-Form, denn es löste sich in einer kalten Lösung von saurem Natriumcarbonat wenigstens teilweise auf, auch war es in Chloroform sehr leicht löslich.

$10,7 \mathrm{~g}$ einer Chloroformlösung enthielten 0,0889 g Farbstoff. Löslichkeit $1: 121$.

\section{Die Esterifizierung des Bilirubins}

geht nach meinen bisherigen Untersuchungen schwieriger vonstatten als die des Hämatoporphyrins. Zwar wirkt auch hier eine einprozentige Salzsäure bereits esterifizierend, doch entsteht ein Gemisch, dem Soda nicht methylierte Substanz entzieht. Erst fünf bis zehn Prozent Salzsäure haltender Methylalkohol liefert ein sodafestes Produkt. Von meinen Versuchen schildere ich den folgenden:

$2 \mathrm{~g}$ Bilirubin $^{1}$ ) wurden durch $100 \mathrm{ccm} 90 \%$ igen Methylalkohol und $15 \mathrm{ccm}$ methylalkoholisches Ammoniak ${ }^{2}$ ) in Lösung

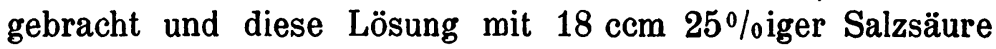
versetzt, so daß eine in bezug auf Chlorwasserstoff $3 \%$ ige Lösung vorlag, die das Bilirubin in feiner Verteilung enthielt. Beim kurzen Erwärmen ging es in Lösung, doch wurde im ganzen zwei Stunden am Rückflußkühler erhitzt, worauf der Methylalkohol abdestilliert wurde. Der Rückstand wurde chlorfrei gewaschen, auf Fließpapier und dann im Vakuum getrocknet. Da eine Probe dieses Produktes aber durch warme Sodalösung allmählich in Lösung ging, wurde der Rest in Chloroform gelöst und der Lösung $20 \mathrm{ccm}$ Methylalkohol und $8 \mathrm{ccm} \mathrm{25 \%} \%$ ige Salzsäure zugefügt, worauf sie 10 Minuten lang im Sieden erhalten wurde. Nach Zusatz von Wasser wurde die Chloroformschicht getrennt, noch einmal mit Wasser und dann mit verdünnter Sodalösung behandelt, bis letztere sich gegeben.

1) Die Analyse dieses Präparats ist auf Seite 475, Anm. 1, an-

2) $1 \mathrm{ccm}$ derselben entsprach $2,9 \mathrm{ccm} \mathrm{n}-\mathrm{NH}_{3}$, so daß also $33,5 \mathrm{n}-\mathrm{NH}_{3}$ angewendet worden sind, d. h. aber fast 10 Moleküle Ammoniak auf 1 Molekül Bilirubin. Auch in wässeriger Lösung braucht man weit mehr als die berechnete Menge Ammoniak, Bilirubin als zweibasisch angenommen. 
nicht mehr färbte, ${ }^{1}$ ) worauf getrocknet und das Chloroform bis auf einen kleinen Rest abdestilliert wurde. Dieser wurde durch Äther gefällt, der entstandene Niederschlag abgesaugt, mit Äther nachgewaschen ${ }^{2}$ ) und im Vakuum getrocknet.

Dieser aus Eisessig wenn auch nur undeutlich krystallisierende Körper stellt ein schwarzgrünes Pulver vor, das von Sodalösung auch in der Wärme nicht angegriffen wird, auch kalte Natronlauge ist ohne Einfluß, beim Erwärmen geht Substanz in Lösung. Aus letzterer fällen Säuren einen in Chloroform mit grüner Farbe löslichen Körper. Die Gmelinsche und die Ehrlichsche Diazoreaktion fallen mit dem Ester positiv aus, dagegen versagt die noch bei Spuren von Bilirubin kenntliche Kondensation mit Formaldehyd unter dem Einfluß konzentrierter Schwefelsäure, die sich hier durch eine intensive Blaufärbung bemerkbar macht. Die Ausbeute betrug etwa 1,5 g.

Die Analyse gab folgendes Resultat:

I. 0,1382 Subst. $\left(120^{\circ}\right): 0,3348 \mathrm{CO}_{2}$ und $0,0790 \mathrm{H}_{2} \mathrm{O}$. $0,1981 \gg\left(110^{\circ}\right): 0,1273 \mathrm{AgJ}$ (Zeisel).

Bei anderen Darstellungen, die ähnlichen Verlauf genommen hatten, aber mit weniger reinem Bilirubin ausgeführt worden waren, geben die sodafesten Anteile auch ähnliche Werte.

II. 0,1053 Subst. $\left(110^{\prime \prime}\right): 0,2535 \mathrm{CO}_{2}$ und $0,0598 \mathrm{H}_{2} \mathrm{O}$.

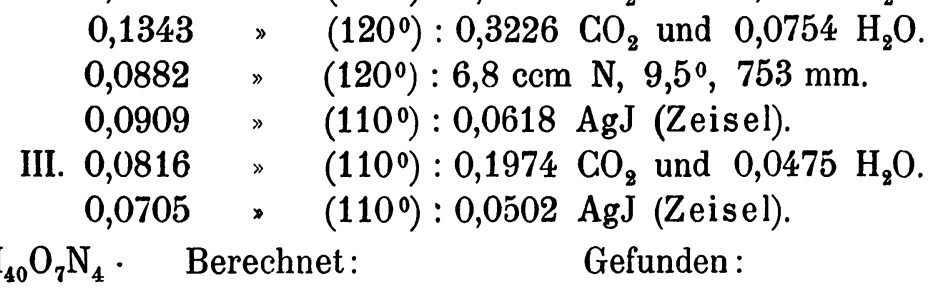

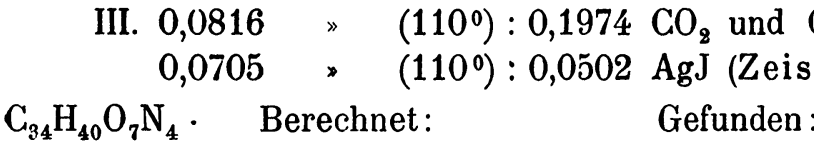

III. $\begin{array}{r}0,0816 \\ 0,0705\end{array}$ » $\left(110^{\circ}\right): 0,1974 \mathrm{CO}_{2}$ und $0,0,0502 \mathrm{AgJ}$ (Zeis
${ }_{40} \mathrm{O}_{7} \mathrm{~N}_{4} \cdot$ Berechnet:

$\begin{array}{cccccc} & & \text { I } & \text { II } & \text { III } \\ \% \mathrm{C} & 66,23 & 66,07 & 65,66 & 65,51 & 65,98 \\ \% \mathrm{H} & 6,49 & 6,36 & 6,3 & 6,24 & 6,51 \\ \% \mathrm{~N} & 9,9 & & 9,16 & & \\ 2 \mathrm{CH}_{3} \% & 4,87 & 4,1 & 4,34 & & 4,54\end{array}$

1) Die Soda nahm sehr geringe Mengen scheinbar unveränderten Bilirubins fort: die Lösung war orange gefärbt, die aus dieser Lösung durch Schwefelsäure gefällte Substanz enthielt kein Methyl.

2) Hierbei bleibt Substanz in Lösung. 
Neben dieser Formel kommt auch die um zwei Wasserstoff-Atome ärmere in Betracht, welche darauf schließen ließe, daß eine Oxydation durch den Sauerstoff der Luft eingetreten wäre. Zur Sicherstellung des Resultats, daß obiger Körper sich durch einmalige Methylierung und einmalige Einlagerung von Methylalkohol aus Bilirubin gebildet hat, soll daher der MethylierungsprozeB unter Abschluß von Luft wiederholt werden. Auch sind Versuche in Angriff genommen worden, das Bilirubin in alkalischer Lösung durch Methylsulfat zu methylieren.

Ich bitte daher ausdrücklich darum, mir die Alkylierung des Bilirubins überlassen zu wollen.

\section{Die Oxydation des Bilirubins.}

Die erste Beobachtung vom Auftreten des «Imids bei der Oxydation des Bilirubins machte Herr Schmiedel in meinem Institut. Ich schildere den Versuch nach den Angaben in der Dissertation des Genannten.

$4 \mathrm{~g} \beta$-Bilirubin $^{1}$ ) wurden in $100 \mathrm{ccm} 50 \%$ iger Schwefelsäure dadurch gelöst, daß kleine Mengen des Farbstoffs auf eine breite Fläche der Säure geschichtet wurden, das Eintragen weiterer Mengen wurde erst fortgesetzt, wenn vollständige Lösung der ersten Anteile eingetreten war. ${ }^{2}$ )

Die erhaltene schwefelsaure Lösung wurde auf $0^{\circ}$ abgekühlt und unter Turbinieren tropfenweise die Lösung von $10 \mathrm{~g}$ Chromsäure in $20 \mathrm{ccm}$ Wasser zugegeben, während welcher Operation die Temperatur nicht über $3^{\circ}$ stieg, sodaß das Eintropfen etwa 11/8 Stunden in Anspruch nahm. Die stark schäumende olivgrün gefärbte Flüssigkeit wurde dann noch eine weitere Stunde turbiniert, schließlich erschöpfend mit Äther ausgezogen und der ätherischen Lösung die sauren Körper durch eine verdünnte Sodalösung entzogen, worauf sie

1) $\beta$-Bilirubin ist der Anteil des rohen Bilirubins, der nach der Extraktion der Gallensteine im Chloroform gelöst bleibt und durch Fällen mit Alkohol isoliert wurde.

2) Beim Anreiben von Bilirubin mit Schwefelsäure entzieht sich immer ein Teil der Lösung, dadurch daß er in einen harzigen Kuchen übergeht. 
mit Natriumsulfat getrocknet wurde. Nachdem dann der Äther abdestilliert worden war, hinterblieb ein brauner, stechend riechender Sirup. Dieser wurde zunächst über Natronkalk im Vakuum getrocknet, wonach der stechende Geruch verschwunden war, die nunmehr wachsartige Masse zeigte bereits den charakteristischen Geruch nach dem "Imid». Zur Reinigung wurde sie mit 40\% Wasser gewaschene und getrocknete ätherische Lösung vorsichtig verdunstet. Es hinterblieben nadelförmige Krystalle, die den charakteristischen Geruch des Methyläthylmaleinimids zeigten. Leider reichte ihre Menge nicht einmal zu einer Bestimmung des Schmelzpunktes hin.

Aus den in der Sodalösung verbliebenen sauren Anteilen konnte Hämatinsäure (es wurden $0,87 \mathrm{~g}$ des charakteristischen Calciumsalzes der Säure $\mathrm{C}_{8} \mathrm{H}_{8} \mathrm{O}_{5}$ ) und Bernsteinsäure (Schmelzpunkt $182^{\circ}$ ) isoliert werden. Der so wichtige Befund, daß bei der Oxydation eines Bilirubinpräparates das "Imid" entsteht, soll wiederholt werden, sobald mir einmal wieder größere Mengen von Bilirubin zur Verfügung stehen. Leider bin ich in den letzten Jahren von meinem Hauptlieferanten in Stich gelassen worden.

Das Auftreten des «Imids» wurde alsdann auch noch von mir bei der Oxydation des Bilirubins in alkalischer Lösung beobachtet.

$10 \mathrm{~g}$ Rohbilirubin wurden mit Hilfe von $105 \mathrm{ccm} 15 \%$ iger Kalilauge (also etwa 14 Moleküle $\mathrm{KOH}$ ) zum Liter gelöst und diese Lösung nach zweitägigem Stehen auf die Hälfte eingedampft, wonach $30 \mathrm{ccm} \mathrm{3 \%}$ iger Wasserstoffsuperoydlösung zugefügt wurden ( $1^{1 / 2}$ Atome 0$)$. Alsdann wurde durch verdünnte Schwefelsäure vorsichtig sauer gemacht, wobei sich ein sehr charakteristischer Geruch bemerkbar machte und ein Niederschlag auftrat, von dem filtriert wurde.

\section{Filtrat.}

A. Das Filtrat wird erschöpfend ausgeäthert, worauf es nicht mehr riecht, der ätherische Auszug wird mit Sodalösung geschüttelt, die den riechenden Körper fortnimmt. 
a) Die verbleibende ätherische Lösung wird zu weiteren Ausschüttelungen verwendet.

b) Die Sodalösung wird durch 0,1\% ige Kaliumpermanganatlösung oxydiert, bis eine Probe beim Ansäuern nicht mehr den eigentümlichen Geruch gibt, dann angesäuert und ausgeäthert. Der Äther hinterläßt typisch krystallisierte Hämatinsäure $\mathrm{C}_{8} \mathrm{H}_{8} \mathrm{O}_{5}$. Der riechende Körper stellt also eine Vorstufe dieser Säure vor. Analyse des Kalksalzes der Hämatinsäuren

$$
0,1104 \mathrm{~g}: 0,0311 \mathrm{CaO}=20,14 \% \text { Ca. }
$$

B. Das erschöpfend ausgeätherte Filtrat wird wieder alkalisch gemacht und dann stark eingedampft, beim Wiederansäuern tritt kein Geruch mehr auf. Die mit Äther hergestellten Auszüge werden wieder mit Sodalösung behandelt.

a) Die verbleibende ätherische Lösung wird zu weiteren Ausschüttelungen verwendet.

b) Die Sodalösung wird angesäuert, wobei jetzt der typische Geruch nach bisubstituierten Maleinsäuren auftritt. Es wird mit Wasserdämpfen destilliert, wonach sich zeigt, daß $\alpha$ ) eine ätherlösliche Säure höchstens spurenweise zurückgeblieben ist.

B) Das Destillat wird mit Barytwasser alkalisch gemacht und stark eingedampft, der Rückstand mit Salzsäure angesäuert und die saure Lösung wieder ausgeäthert. Die ätherische Lösung wird mit Kalkwasser ausgeschüttelt, der Überschuß des Ca durch Einleiten von Kohlensäure entfernt, das Filtrat vom Calciumcarbonat eingedampft und der Rückstand mit wenig Wasser aufgenommen. Da diese Lösung beim Erhitzen keine Ausscheidung gab, wurde sie mit Alkohol gefällt und der Niederschlag im Vakuum getrocknet.

$0,0554 \mathrm{~g}$ Substanz $\left(110^{\circ}\right): 0,0323 \mathrm{~g} \mathrm{CaSO}_{4}$.

Ber. für: $\mathrm{C}_{7} \mathrm{H}_{8} \mathrm{CaO}_{4}+\mathrm{H}_{2} \mathrm{O}: \%$ Ca 18,7. Gef.: 17,1.

Niederschlag. Ihm haftet ein charakteristischer Geruch an. Er wird daher in eine Kochflasche gespült, mit Äther überschichtet und die Schichten zur Vermeidung von Emulsionen nur sanft bewegt, der Äther wird dann abgegossen und die Behandlung mit Äther öfters wiederholt. Die ätherischen Lösungen werden sodann mit Sodalösung extrahiert und getrocknet. Nach 
Abdestillation des Äthers verbleibt das typisch riechende Imid und zwar erscheint ein Uhrglas von $5 \mathrm{~cm}$ Durchmesser von den Krystallen überzogen. Leider reichte die Menge, da die Substanz noch nicht ganz rein war, auch hier nicht zur näheren Charakterisierung hin.

Aus der Sodalösung wurde dann auf die bereits angegebene Weise noch etwas Hämatinsäure isoliert.

Nachdem, wie soeben geschildert, dem Niederschlag die noch anhängenden ätherlöslichen Teile entzogen worden waren, wurde er erneut in einem Liter 1,5\% iger Kalilauge gelöst, der Äther durch Erwärmen entfernt, und in die auf $3^{\circ} \mathrm{ab}$ gekühlte Lösung $310 \mathrm{ccm}$ einer $3,1 \%$ igen Lösung von Kaliumpermanganat eingetragen, die gleichfalls abgekühlt worden war. Diese Operation, die unter Turbinieren durchgeführt wurde, nahm etwa eine Stunde in Anspruch, worauf vom Manganschlamm abfiltriert und dieser Niederschlag ausgewaschen wurde. Da das alkalische Filtrat an Äther nichts abgab, wurde angesäuert, ein hierbei entstehender bedeutender Niederschlag - er wog nach dem Trocknen $5 \mathrm{~g}$ - abfiltriert und das Filtrat ausgeäthert. Nach Abdestillation des Äthers verblieb ein Rückstand, der, nach dem typischen Geruch zu urteilen, eine bisubstituierte Maleinsäure enthalten mußte. Etwas größere Mengen derselben wurden noch erhalten, nachdem die ausgeätherte saure Flüssigkeit, wieder alkalisch gemacht, auf ein kleines Volumen konzentriert und nach dem Ansäuern erneut ausgeäthert worden war. Aus diesen Ätherrückständen wurden dann auf die bereits angegebene Weise Baryumsalze hergestellt. Die Lösung derselben in kaltem Wasser schied beim Erhitzen ein hierbei schwer löslich werdendes Salz ab, allerdings wurden keine charakteristisch ausgebildeten Krystalle erhalten.

0,0902 g Substanz $\left(100^{\circ}\right): 0,0660 \mathrm{~g} \mathrm{BaSO}_{4}$.

Ber. für: $\mathrm{C}_{7} \mathrm{H}_{8} \mathrm{O}_{4} \mathrm{Ba}+\mathrm{H}_{2} \mathrm{O}^{\circ}: \% \cdot \mathrm{Ba} 44,05$. Gef.: 43,1 .

Ein anderer Teil wurde aus der wässerigen Lösung durch Alkohol gefällt, er wurde nach dem Trocknen als ein gelbliches Pulver erhalten.

0,2494 g Substanz $\left(110^{\circ}\right): 0,1866 \mathrm{Ba}=44,04 \% \mathrm{Ba}$. 
Beim Auflösen beider Salze in verdünnter Salzsäure entwickelte sich ein starker Geruch nach der bisubstituierten Maleinsäure. Es ist nach allem das Auftreten des Methyläthylmaleinsäureanhydrids bei der 0xydation des Bilirubins in alkalischer Lösung zwar nicht sicher bewiesen, aber höchst wahrscheinlich gemacht. Es muß aber der Komplex des Bilirubins, der das Imid resp. Anhydrid der Methyläthylmaleinsäure liefern kann, in anderer Weise als im Hämatin dem großen Verbande einverleibt sein, so daß er nur schwierig abzutrennen ist, woraus sich dann die sehr geringen Ausbeuten an dem gesuchten Körper erklären.

Herrn Prof. Windaus bin ich zu großem Danke verpflichtet für Überlassung von Rückständen aus menschlichen Gallensteinen, aus denen ich Bilirubin gewinnen konnte. Ich möchte ihm auch an dieser Stelle herzlichst danken.

Stuttgart, am 23. November 1912. 